\title{
A Watermarking Algorithm Based on Wavelet and Hadamard Transform for Color Image
}

\section{Hongshou Yan, Weimin Yang}

College of Computer and Information Engineering, Central South University of Forestry \& Technology, Changsha 410004, Hunan, China.

Email: 165393586@qq.com, luan_yang@126.com

Received 2013

\begin{abstract}
Digital image watermarking is a useful solution to the problem of information security, copyright and network security. In this paper, we propose a watermarking algorithm for color image based HT and DWT. A binary image as watermark is embedded into green component or blue component of color image. The algorithm can satisfy the transparence and robustness of the watermarking system very well. The experiment based on this algorithm demonstrates that the watermarking is robust to the common signal processing techniques including JPEG compressing, adding noise, low pass filter, and mosaic.
\end{abstract}

Keywords: Digital Watermarking; Hadamard Transform(HT); Discrete Wavelet Transform(DWT); Color Image; Copyright Protection

\section{Introduction}

Today, as the digitization develops day by day, the protection of digital information becomes an urgent problem. In order to resist different kinds of infringement, a new technology that called watermarking had been put forward to in the international scope. Watermark is sequence carrying information about the copyright owner to embed into the digital image [1], audios and videos in order that owners can read it out while unauthorized users cannot 1 easily remove it.

There are many methods to embed the watermark. It can be divided into two classes: spatial-domain watermarks and transform-domain watermarks. The spatial domain is so simple that the watermark can be damaged easily, but the transform-domain algorithm can be resist intensity attack, watermark information can't be damaged easily. The transform algorithm includes chiefly DWT, DFT and DCT[2,3,4]. Wavelet transform is superior to time-frequency transform for its inner predominance. For example, wavelet has the character of multi-resolution, which can avoid the rectangle brought by DCT. In fact, it has more application fields in engineering and computer science. In this paper, a new blind watermarking algorithm that embeds a meaningful binary image into the color images is proposed based HT-DWT according to HT and DWT characteristics.

The rest of the paper is organized as follows. Section 2 introduces the hadamard transform and discrete wavelet transforms analysis in briefly. In section 3, a new blind watermark algorithm for color image based HT-DWT domain is presented in detail. Experimental results are described in section 4. Finally, in section 5 the conclusion is given.

\section{Watermark Embedding Principles}

\subsection{Hadamard Transform Analysis}

Hadamard transform by two values, namely 1 and -1 ,as a basic function expand made that it satisfies the complete orthogonal. Hadamard function is binary orthogonal functional corresponding to the two states in digital logic, and therefore more suitable for image processing hardware to achieve a faster rate than other transform. It has been widely used in the area of image processing and image compression. Dimensional discrete Hadamard transform positive transform and inverse transform, such as the definition of formula (1) and (2) [5]:

$$
\begin{aligned}
& H(u, v)=\frac{1}{N} \sum_{x=0}^{N-1} \sum_{y=0}^{N-1} f(x, y)(-1)^{\sum_{i=0}^{N-1}\left(b_{i}(x) b_{i}(u)+b_{i}(y) b_{i}(v)\right)} \\
& f(x, y)=\frac{1}{N} \sum_{u=0}^{N-1} \sum_{v=0}^{N-1} H(u, v)(-1) \sum_{i=0}^{N-1}\left(b_{i}(x) b_{i}(u)+b_{i}(y) b_{i}(v)\right)
\end{aligned}
$$

$H(0,0)$ is called image block the DC component hadamard transform domain. Using an interactive relationship can generate higher order transform matrix of Ha- 
damard transform ,such as the formula (3) below.

$$
H_{2}=\left[\begin{array}{cc}
+1 & +1 \\
+ & -1
\end{array}\right], H_{2^{k+1}}=\left[\begin{array}{ll}
H_{2^{k}} & H_{2^{k}} \\
H_{2^{k}} & H_{2^{k}}
\end{array}\right], k=1,2,3, \ldots
$$

\subsection{Discret Wavelet Transform Principles}

Wavelet transform is a time-frequency domain combined analysis method. It has multi-resolution analysis features. Each level of the wavelet decomposition has four sub-images with same size. Let the $L L_{k}$ stands for the approximation sub-image and $L H_{k}, H L_{k}, H H_{k}$ stand for the horizontal, vertical and diagonal direction highfrequency detail sub-image respectively. Where the variable $k=1,2,3, \ldots(k \in N)$ is the scale or the level of the wavelet decomposition.

After wavelet decomposition, many signal processing, such as compression and filter are likely to change the high-frequency wavelet coefficients. If the watermark sequence is embedded into this part, its information may be lost in the processing in sequence, which will reduce the robustness of the watermark [3]. In order to ensure the watermark has a better imperceptibility and robustness, the approximation sub-image $L L_{3}$ coefficients are chosen to embed watermark. We can achieve the transform of the separable wavelet as in Figure 1.

\section{Proposed Watermarking Algorithm}

Here, the readable watermark is a $q \times q$ binary image. We arrange the binary image to 0,1 watermark sequence $w m$. And the length of wm is the $p \times q$. Original image is a $m \times n$ color image.

\subsection{Watermark Embedding Scheme}

Step1 `A one-dimension chaotic sequence is originated from a logistic mapping $X_{n+1}=u X_{n}\left(1-X_{n}\right)$ [4]. The sequence has the same size as the length of the wm. Apply a threshold value, and then get $0-1$ sequences $A^{*}$. The program performs a XOR operation of this wm with the binary watermark image. $X_{0}$ and $u$ are password. The sequence of the binary watermark image after encrypting is:

\begin{tabular}{|c|c|c|c|}
\hline $\mathrm{LL}_{3}$ & $\mathrm{LH}_{3}$ & $\mathrm{LH}_{2}$ & \\
\cline { 1 - 2 } $\mathrm{HL}_{3}$ & $\mathrm{HH}_{3}$ & & \\
\cline { 1 - 2 } $\mathrm{HL}_{2}$ & $\mathrm{HH}_{2}$ & \\
\cline { 1 - 2 } $\mathrm{HL}_{1}$ & & \\
\hline
\end{tabular}

Figure 1. Three level wavelet decomposition.

$$
W=X O R\left(A^{*}, w m\right)=\{w(i) \in\{0,1\} \mid 1=<i<=p \times q\}
$$

Step2 Extracting the green components $(G)$ and the from original color image. It is divided into square blocks of size $8 \times 8$ pixels. Then the HT is applied in each block. Then the DC value $H_{i, j}(1,1)$ of each block is collected together to get a new matrix $I$.

$$
I=\left[\begin{array}{ccccc}
H_{1,1}(1,1) & H_{1,2}(1,1) & \ldots & H_{1, k 1-1}(1,1) & H_{1, k 1}(1,1) \\
H_{2,1}(1,1) & H_{2,2}(1,1) & \ldots & H_{2, k 1-1}(1,1) & H_{2, k 1}(1,1) \\
\ldots & \ldots & \ldots & \ldots & \ldots \\
H_{k 2-1,1}(1,1) & H_{k 2-1,2}(1,1) & \ldots & H_{k 2-1, k 1-1}(1,1) & H_{k 2-1, k 1}(1,1) \\
H_{k 2,1}(1,1) & H_{k 2,2}(1,1) & \ldots & H_{k 2, k 1-1}(1,1) & H_{k 2, k 1}(1,1)
\end{array}\right]
$$

where, $k 1=n / 8, k 2=m / 8$

Step3 Make the new matrix $I$ to do a one-scale two-dimension discrete wavelet transform with haar. According to quantization step value s, make the low coefficient LL to qualified adjustment, then embed the watermark value. The detailed process is as follows:

The quantified value $q(i, j)$ of the low-frequency wavelet coefficient can be obtained by:

$$
q(i, j)=\lfloor L L(i, j) / s\rfloor
$$

The process of embedding watermark information is as follows:

If $\bmod (q(i, j), 2)==W(k)$, adjust the low-frequency wavelet coefficient to

$$
L L^{\prime}(i, j)=q(i, j) \times s+s / 2
$$

If $\bmod (q(i, j), 2) \neq W(k)$, adjust the low-frequency wavelet coefficient to

$$
\text { If } L L(i, j)-q(i, j) \times s \in(0, s / 2)
$$

then $L L^{\prime}(i, j)=(q(i, j)-1) \times s+s / 2$

else $L L^{\prime}(i, j)=(q(i, j)+1) \times s+s / 2$

where, $i=1,2, \ldots, m / 16, j=1,2, \ldots, n / 16, k=1,2,3, \ldots, p \times q$.

Step 4 Make wavelet inverse transform.

Step 5 The $H_{i j}(1,1)$ of each block can be obtained by extracting the corresponding value the wavelet inversetransform matrix, then make HT inverse-transform each sub-block. Changing the double-precision real number to unsigned 8-bit integer. Thus, obtain the color components in which watermark are embedded. Finally, we transform the image from three-basic-color image into true color RGB space. Then we will get the watermarked color image.

\subsection{Watermark Extracting Scheme}

The processes of watermark extracting and embedding are reverse. When extracting watermark, the detailed ways is as follows:

Step1 Extracting the green components (G), it is divided into $8 \times 8$ sub-block. Then the HT is applied in each block. Then the DC value $H_{i, j}(1,1)$ of each block 
is collected together to get a new matrix $I^{\prime}$. $i=1,2, \ldots, m / 8, j=1,2, \ldots, n / 8$.

$I^{\prime}=\left[\begin{array}{ccccc}H_{1,1}^{\prime}(1,1) & H_{1,2}^{\prime}(1,1) & \ldots & H_{1, k 1-1}^{\prime}(1,1) & H_{1, k 1}^{\prime}(1,1) \\ H^{\prime}{ }_{2,1}(1,1) & H^{\prime}{ }_{2,2}(1,1) & \ldots & H^{\prime}{ }_{2, k 1-1}(1,1) & H_{2, k 1}^{\prime}(1,1) \\ \ldots & \ldots & \ldots & \ldots & \ldots \\ H_{k 2-1}^{\prime}{ }^{\prime}(1,1) & H^{\prime}{ }_{k 2-1,2}(1,1) & \ldots & H^{\prime}{ }_{k 2-1, k 1-1}(1,1) & H^{\prime}{ }_{k 2-1, k 1}(1,1) \\ H^{\prime}{ }_{k 2 k, 1}(1,1) & H^{\prime}{ }_{k 2,2}(1,1) & \ldots & H_{k 2, k 1-1}(1,1) & H^{\prime}{ }_{k 2, k 1}(1,1)\end{array}\right]$

where, $k 1=n / 8, k 2=m / 8$.

Step2 Make the matrix $I^{\prime}$ to do a one-scale two-dimension discrete wavelet transform with haar, and extract the watermark from low-frequency wavelet coefficient LL. The detailed way is as follows:

$$
\begin{aligned}
q(i, j) & =\lfloor L L(i, j) / s\rfloor \\
W^{\prime}(k) & =\bmod (q(i, j), 2)
\end{aligned}
$$

where, $i=1,2, \ldots, m / 16, j=1,2, \ldots, n / 16, k=1,2,3, \ldots, p \times q$.

The word $s$ refers to quantization step value, and $W^{\prime}(k)$ refers to extracted watermark sequences.

Step3 The watermark sequences which is extracted carry on chaotically decryption. Then it can be transformed into a binary image.

Here we use the normalized correlation (NC) to measure the similarity between original image $W$ and the detected watermark image $W^{\prime}[6]$.

$$
N C=\frac{\sum_{I=1}^{n} \sum_{J=1}^{n} W(i, j) \cdot W^{\prime}(i, j)}{\sum_{i=1}^{N} \sum_{j=1}^{N} W(i, j) \cdot W(i, j)}
$$

In order to get rid of the impact of subjective factor, this paper adopts peak signal-to-noise ratio (PSNR) to measure the fidelity between the original image and the image which watermark is embedded.

\section{Experiment Results}

In this paper, $400 \times 512 \times 3$ true color lena image and baboon image are selected as the original image and a $20 \times 40$ binary image is selected as the watermark image. Lena image is embedded watermark in the blue components after contrasting the green components with the blue components. The quantization step value $s$ is 106 in lena image.

Watermark image is embedded into the blue components of lena image. The PSNR value of watermarked image (Figure 2(c)) is 42.05, the $N C$ value of extracted watermark (Figure 2(d)) is 1.0. From Figure 2, the human eye may feel no difference between the original image and the watermarked image. The algorithm can satisfy the transparence.

In order to investigate the robustness of the watermarking scheme, the watermarked image was attacked by various signals processing technique, such as JPEG compression, Additive gaussian noise, Additive salt noise, Median filtering, image enhancement, mosaic and other kinds of image processing approaches to attack the watermarked image. Table 1 show the results of our simulations and Figure $\mathbf{3}$ shows that it be extracted watermark from being attacked watermarked lena image.

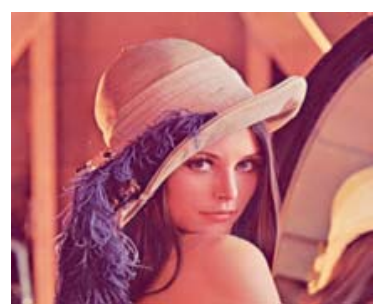

(a)

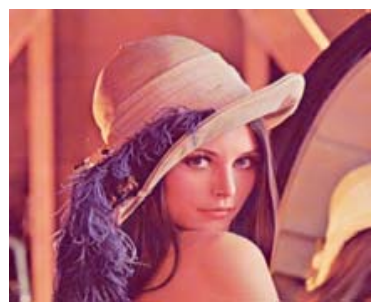

(c)
JLU

(b)

\section{JLU}

(d)
Figure 2. (a) original lena image ; (b) original watermark ;

\begin{tabular}{|c|c|c|c|}
\hline \multirow{2}{*}{ Test Method } & \multicolumn{3}{|c|}{ lena } \\
\hline & parameter & PSNR & $N C$ \\
\hline \multirow{3}{*}{$\begin{array}{c}\text { JPEG } \\
\text { compression }\end{array}$} & $\mathrm{Q}=80$ & 32.68 & 1.0000 \\
\hline & $\mathrm{Q}=50$ & 31.23 & 0.9936 \\
\hline & $\mathrm{Q}=30$ & 30.01 & 0.9488 \\
\hline \multirow{4}{*}{ Noise adding } & Salt\&pepper 1\% & 26.06 & 0.9981 \\
\hline & Salt\&pepper 2\% & 23.15 & 0.9855 \\
\hline & Speckle noise 0.02 & 22.41 & 0.9976 \\
\hline & Gaussian noise 0.01 & 21.19 & 0.9617 \\
\hline \multirow{3}{*}{ Filtering } & Gaussian lowpass $(3 \times 3)$ & 32.01 & 1.0000 \\
\hline & Median filtering $(2 \times 2)$ & 28.62 & 0.9272 \\
\hline & Median filtering $(3 \times 3)$ & 31.84 & 0.9939 \\
\hline \multirow{3}{*}{$\begin{array}{c}\text { image } \\
\text { enhancement }\end{array}$} & Edge sharppen & 28.72 & 1.0000 \\
\hline & Gaussian blurring & 31.80 & 1.0000 \\
\hline & Moving blurring & 27.10 & 0.9607 \\
\hline \multirow{3}{*}{ Image mosaic } & $2 \times 2$ & 31.36 & 1.0000 \\
\hline & $3 \times 3$ & 28.04 & 1.0000 \\
\hline & $4 \times 4$ & 27.18 & 1.0000 \\
\hline
\end{tabular}
(c) watermarked image; (d) extracted watermark.

Table 1. Test results of watermarked image. 
JE:

(a)

\section{DLW}

(c)

\section{dLU}

(d)

\section{JES:}

(e)

Figure 3. (a) JPEG compression $Q=30$; (b) Salt \&pepper noise 2\%; (c) Gaussian noise 0.01; (d) Median filtering 2×2; (e) Moving blurring.

\section{Conclusions}

In this paper, a new blind technique for embedding a binary image into color digital image based on HT and DWT has been proposed, which is robust to the common signal processing techniques including JPEG compressing, noise, low pass filter, median filter, image enhance and mosaic. The algorithm is not only simply but also valid. This blind watermarking algorithm can broaden its application area.

\section{Acknowledgment}

This work was supported by the Science and technology projects in Hunan Province(No.2010TZ4012)

\section{REFERENCES}

[1] I.J.Cox, J.Kilian, F.T.Leighton, and T.Shamoon, "Secure spread spectrum watermarking for multimedia”, IEEE Trans. Image Processing, vol .6, pp. 1673-1687, December 1997.

[2] G.Langelaarand, R.Lagendijk, "Optimal differential energy watermarking of DCT encoded images and video", IEEE Trans., vol .10,pp.148-158,January 2001.

[3] J.W.Huang, Yun Q.SHI, W.D.Cheng, “Image watermarking in DCT: an embedding strategy and algorithm” ,Journal of Electronic, vol .28,pp.57-60, April 2000

[4] Z.M.Zhang, L.Wang, "Semiblind image watermarking algorithm in DCT domain with chaotic encryption", Computer Engineering, vol.29,pp. 9-11, October 2003.

[5] Pankaj U.Lande, Sanjay N.Talbar., "FPGA Implementation of Adaptive Watermarking Using Human Visual Model”,.ICGST-PDCS Journal, vol.9,pp.17-21,January 2009

[6] L.C.Li, Z.Y.Lu, "Desynchronization attack on digital watermarks and their counte measures,” Journal of image and graphics, vol .10,pp.403-409, October 2005. 